\title{
Conformational Dynamics Contribute to Substrate Selectivity and Catalysis in Human Kynureninase
}

Christos S. Karamitros ${ }^{1 \pi \square}$, Kyle Murray ${ }^{2 \pi}$, Yusuke Sugiyama ${ }^{3}$, Yoichi Kumada ${ }^{3}$, Kenneth A. Johnson $^{4}$, George Georgiou ${ }^{1,4,5,6}$, Sheena D'Arcy ${ }^{*}$ and Everett M. Stone $e^{4,6^{*}}$

${ }^{1}$ Department of Chemical Engineering, The University of Texas at Austin, Austin, TX 78712, United States.

${ }^{2}$ Department of Chemistry and Biochemistry, The University of Texas at Dallas, Richardson TX 75080 United States.

${ }^{3}$ Department of Molecular Chemistry and Engineering, Graduate School of Science and Technology, Kyoto Institute of Technology, Kyoto, Japan

${ }^{4}$ Department of Molecular Biosciences, The University of Texas at Austin, Austin, TX 78712, United States.

${ }^{5}$ Institute for Cellular and Molecular Biology, The University of Texas at Austin, Austin, TX 78712, United States.

${ }^{6}$ Department of Oncology, University of Texas Dell Medical School, LiveSTRONG Cancer Institutes, Austin, Texas, United States.

$\square$ Current Address: Aeglea BioTherapeutics, 805 Las Cimas Parkway, Austin, TX 78746

*Correspondence to: Sheena D'Arcy and Everett Stone

E-mails: sheena.darcy@utdallas.edu and stonesci@utexas.edu

Keywords: enzyme catalysis, enzyme kinetics, enzyme mechanism, hydrogen-deuterium exchange, conformational change 
Table of Contents

1. Methods

2. SI Tables

S1. Burst experiment parameters of HsKYNase-OH-KYN and PfKYNase-KYN reactions.

S2. Statistical analysis of pre-steady-state data fitting of HsKYNase-OH-KYN

S3. Statistical analysis of pre-steady-state data fitting of PfKYNase-KYN

S4 \& S5. Experimental parameters and raw data of HDX-MS experimental runs.

S6. Composition of main fed-batch culture medium

S7. Composition of fed-batch feeding medium

3. SI Schemes

S1. Catalytic mechanism of KYNases

\section{SI Figures}

S1. Steady-state kinetic plots in $\mathrm{H}_{2} \mathrm{O}$

S2. AA sequence alignment of HsKYNase vs PfKYNase

S3. Excitation/Emission spectra of $\mathrm{AA}$ and $\mathrm{OH}-\mathrm{AA}$

S4. Confidence contour analysis of HsKYNase pre-steady state analysis

S5. Confidence contour analysis of PfKYNase pre-steady state analysis

S6. Free energy profile of HsKYNase/PfKYNase with preferred substrate

S7. Peptide coverage map for HsKYNase and PfKYNase

S8. HDX-MS of HsKYNase with OH-KYN or KYN after 100 minutes D exposure

S9. HDX-MS of PfKYNase with KYN or OH-KYN after 100 minutes D exposure

S10. Steady-state kinetic plots in $\mathrm{D}_{2} \mathrm{O}$ 


\section{METHODS}

\section{Expression and purification of PfKYNase.}

Bacterial KYNase from Pseudomonas fluorescens was cloned, expressed and purified as described elsewhere. ${ }^{1}$ Briefly, codon-optimized gene sequence for recombinant expression in E. coli were cloned into pET28a plasmid that confers resistance against kanamycin. The full-length enzyme was expressed in BL21 (DE3) cells with a N-terminal 6-His tag to facilitate purification by immobilized-metal affinity chromatography (IMAC). Typical expression yields were $\sim 200 \mathrm{mg} / \mathrm{L}$ of Terrific Broth medium (TB; Difco, Bectron, Dickinson, and company) for PfKYNase after induction with $0.5 \mathrm{mM}$ Isopropyl $\beta$-D-1thiogalactopyranoside (IPTG, Fisher) at $25{ }^{\circ} \mathrm{C}$. For the affinity purification, $100 \mathrm{mM}$ sodium phosphate, $300 \mathrm{mM} \mathrm{NaCl}$ (Sigma), $20 \mathrm{mM}$ imidazole (Fisher), $1 \mathrm{mM}$ phenylmethylsulfonyl fluoride (PMSF, Sigma), 0.1\% Tween 20 (Sigma), 1 mM pyridoxal-5-phosphate monohydrate (PLP, Fisher), pH 8 was used as lysis and washing buffer whereas the proteins were eluted using the same buffer except for the imidazole which was $300 \mathrm{mM}$. All purification steps were performed at $25^{\circ} \mathrm{C}$ unless otherwise stated. Following elution from the IMAC column the proteins were incubated with $5 \mathrm{mM}$ PLP at $37^{\circ} \mathrm{C}$ for 2 hours in order to saturate the active site with the co-factor and ensure full loading of PLP. Subsequently, the enzymes were buffer exchanged against Dulbecco's phosphate-buffered saline, $\mathrm{pH} 7.4$ (measured at $25^{\circ} \mathrm{C}$ ) using a Vivaspin TURBO 15 ultrafiltration tube (Sartorius, Goettingen, Germany) with a $10 \mathrm{kDa}$ MWCO. The process was repeated at least 3 times until all the excess of PLP was removed. Protein purity was $>95 \%$ as assessed by SDS-PAGE. Fraction of the buffer exchanged protein was subjected to size-exclusion chromatography using a Superdex 200 gel-filtration column attached to an Akta FPLC instrument to ensure the formation of dimers that represent the active oligomeric state of the enzyme. The protein concentration was determined spectrophotometrically at $280 \mathrm{~nm}$ using the predicted molar absorption coefficient based on its primary amino acid sequence assuming that all cysteines are reduced $\left(47043 \mathrm{M}^{-1} \mathrm{~cm}^{-1}\right.$ for PfKYNase and $51340 \mathrm{M}^{-1} \mathrm{~cm}^{-1}$ for HsKYNase). For long-term storage, glycerol was added to a final concentration of $15 \%$ $\mathrm{v} / \mathrm{v}$, and the protein solution was flash frozen in liquid $\mathrm{N}_{2}$ and stored at $-80{ }^{\circ} \mathrm{C}$. Due to very low expression yields of HsKYNase in the conventional shake-flask setup (soluble protein yield $\sim 2 \mathrm{mg} / \mathrm{L}$ TB medium), the enzyme was produced following a fed-batch fermentation process as described in the following section yielding $\sim 1.5 \mathrm{~g} / \mathrm{L}$ of culture. 


\section{Fed-batch fermentation of HsKYNase}

\section{A. Pre-culture}

E. coli $\mathrm{C} 43$ transformed with pMAL vector encoding wild-type HsKYNase was inoculated into a $200 \mathrm{~mL}-$ flask containing $10 \mathrm{~mL}$ of 2xYT medium supplemented with $34 \mathrm{mg} / \mathrm{L}$ chloramphenicol (Cam). The flask was shaken at $225 \mathrm{rpm}$ overnight at $37{ }^{\circ} \mathrm{C}$ and part of the overnight culture was transferred into $100 \mathrm{~mL}$ 2xYT medium supplemented with $34 \mathrm{mg} / \mathrm{L}$ (Cam) (such that the $\mathrm{OD}_{600}$ was 0.1 ) using a $500 \mathrm{~mL}$ baffled flask. The flask was shaken at $200 \mathrm{rpm}$ at $30{ }^{\circ} \mathrm{C}$ until the $\mathrm{OD}_{600}$ value of culture became 1.0.

\section{B. Set-up for main culture}

One liter of culture medium with composition shown in Table S6 was prepared in two L jar fermenter vessel (ABLE/Biott). Feeding medium shown in Table $\mathbf{S 7}$ was also prepared and subsequently the feeding medium was passed through a sterile filter with $0.22 \mu \mathrm{m}$ pores.

\section{Main culture}

All of the pre-culture (approx. $100 \mathrm{~mL}$ ) was transferred to a fermenter, and main culture started with batch operation at $30{ }^{\circ} \mathrm{C}, 600 \mathrm{rpm}$ and 0.9 VVM. Since dissolved oxygen (DO) level in a vessel gradually decreases, agitation speed step-wisely increased up to $1000 \mathrm{rpm}$ to maintain aerobic condition. When the DO level became lower than $5 \%$ of saturation at 1000rpm, it was confirmed that glucose concentration was almost zero by glucose check test. Then, fed-batch culture started with a constant DO level (40\%) using our original PID control system. Agitation speed was kept at $800 \mathrm{rpm}$. IPTG was added to a final concentration of $0.8 \mathrm{mM}$, when the $\mathrm{OD}_{600}$ value of culture reached at 60 . The cultivation continued for approx. $70 \mathrm{~h}$ (total $100 \mathrm{~h}$ after main culture started). Samples (approx. $15 \mathrm{~mL}$ each) were taken with $5 \sim 6 \mathrm{~h}$ intervals. Cultured samples taken were centrifuged at 10,000xg and $4{ }^{\circ} \mathrm{C}$ for $20 \mathrm{~min}$, and the supernatant was removed. The pellets were stored at $-20{ }^{\circ} \mathrm{C}$ and $\mathrm{HsKYNase}$ was purified following the same protocol as described above for PfKYNase. 


\section{Steady-state kinetic analysis of kynureninase enzymes}

The assays were performed in PBS, pH 7.4 at $37^{\circ} \mathrm{C}$ using a BioTek Synergy H1 96-well microplate reader. $\mathrm{KYN}$ and $\mathrm{OH}-\mathrm{KYN}$ concentrations were varied in the range of $0-2 \mathrm{mM}$ in a final volume of $200 \mu \mathrm{L}$ and depending on the enzyme and the substrate being tested for activity, enzyme concentrations in the range of 0.01-0.5 $\mu \mathrm{M}$ were used for steady-state kinetic measurements (typically higher enzyme concentration was used when the non-preferred substrate was studied). The obtained $v /[E]$ (initial velocity/total enzyme concentration) values from the linear region of the reaction progress curves with $<10 \%$ of substrate conversion were plotted against the respective substrate concentrations and the steady-state kinetic parameters $k_{\text {cat }}$ and $k_{\text {cat }} / \mathrm{K}_{\mathrm{M}}$ were calculated by nonlinear regression using the Michaelis-Menten model (equation 1 below) analyzed by the SoftZymics software (Igor Pro, Wavemetrics). In cases where no saturation was observed, the data of the $\mathrm{v} /[\mathrm{E}]$ vs substrate concentration plots were fit to the linear function $\mathrm{y}=\mathrm{a}^{*} \mathrm{x}+\mathrm{b}$, where the slope (a) of the line defines the second order rate constant for substrate binding $k_{\text {cat }} / \mathrm{K}_{\mathrm{M}}$. The molar absorption coefficients of KYN and OH-KYN were used for the conversion of absorbance to substrate concentration ( $4.5 \mathrm{mM}^{-1} \mathrm{~cm}^{-1}$ for KYN and $4.7 \mathrm{mM}^{-1} \mathrm{~cm}^{-1}$ for OH-KYN). Both substrates exhibited a short half-life (primarily OH-KYN) and therefore they were always produced fresh and were kept on ice. It must be emphasized that the substrate $\mathrm{OH}-\mathrm{KYN}$ is commercially provided as a 1:1 racemic mixture of D and $\mathrm{L}$ forms. Therefore, for our $k_{\mathrm{cat}} / \mathrm{K}_{\mathrm{M}}$ and pre-steady state calculations we took into account only the $\mathrm{L}$ form of the substrate. Unless otherwise stated, OH-KYN corresponds to the final actual concentration converted by the enzyme i.e. OH-L-KYN.

For studying solvent isotope effects, steady-state kinetic experiments were performed in PBS which was prepared in $99.9 \%$ deuterium oxide ([ $\mathrm{D}_{2} \mathrm{O}$, Sigma) and the $\mathrm{pD}$ was corrected based on the $\mathrm{pH}$ electrode measurement by adding 0.4 units $\left(\mathrm{pD}=\mathrm{pH}_{\text {read }}+0.4\right)$. For these experiments both substrates were dissolved in $\left[\mathrm{D}_{2} \mathrm{O}-\mathrm{PBS}\right.$, pD $7.4\left(\mathrm{pH} 7.0\right.$ at $\left.25^{\circ} \mathrm{C}\right)$. Subsequently, purified enzyme fractions in $\mathrm{H}_{2} \mathrm{O}-\mathrm{PBS}$ were thawed on ice (taken from $-80{ }^{\circ} \mathrm{C}$ as $15 \% \mathrm{v} / \mathrm{v}$ glycerol stocks) and were added into the $\left[\mathrm{D}_{2} \mathrm{O}-\mathrm{PBS}, \mathrm{pD} 7.4\right.$ containing either KYN or OH-KYN to initiate the reaction. The final dilution of the enzymes in the $\mathrm{D}_{2} \mathrm{O}-\mathrm{PBS}$ was 1:24 to mimic the conditions of the HDX-MS experiments.

$\mathrm{v}=\left(k_{\mathrm{cat}} \times[\mathrm{S}]\right) /\left(\mathrm{K}_{\mathrm{M}}+\mathrm{S}\right)($ equation 1$)$

where $\mathrm{v}$ is the initial velocity of the reaction, $\mathrm{S}$ is the substrate concentration, $k_{\text {cat }}$ is the steady-state maximal rate under saturating substrate concentration and $\mathrm{K}_{M}$ is the substrate concentration at which the enzyme operates at its half velocity. 


\section{Stopped-flow fluorescence spectroscopy for HsKYNase and PfKYNase}

The rate of formation and release of either anthranilate (AA; 1st product of the reaction with $\mathrm{KYN}$ ) or OHanthranilate (OH-AA; 1st product of the reaction with $\mathrm{OH}-\mathrm{KYN}$ ) was followed by stopped-flow fluorescence spectroscopy. The exact excitation and emission wavelengths for AA and OH-AA were determined by recording the excitation and emission spectra for both AA and OH-AA in PBS, pH 7.4 (Figure S3 below) using a fluorospectrometer (Fluorescence System instrument from Photon Technology International). Single pre-steady-state burst measurements were performed by mixing high concentrations of either HsKYNase or PfKYNase (range 5-17.5 $\mu \mathrm{M}$ after mixing) with either $500 \mu \mathrm{M}$ OH-KYN or 800 $\mu \mathrm{M}$ KYN respectively in $\mathrm{PBS}, \mathrm{pH} 7.4$ at $37^{\circ} \mathrm{C}$. The reaction was monitored for a short period of time $(0.25-$ $0.5 \mathrm{~s}$ ) and the fluorescence was excited (slit width was set at $1.24 \mathrm{~mm}$ ) followed by observation of the emitted light using single band bandpass filters (Semrock, Rochester, NY). Traces shown in the main text represent the average of at least 5 different measurements while 2000 data points were collected for each trace.

For the conversion of fluorescence to absolute product concentrations, known concentrations of either AA or OH-AA were mixed 1:1 in the stopped-flow apparatus and the fluorescence signal was recorded for each concentration. Subsequently, the recorded fluorescence signals were plotted against the known concentrations and the generated standard curve served as reference for the conversion. Upon conversion of the fluorescence to absolute product concentrations, the averaged pre-steady state burst traces ( 5 traces per concentration) were fit to single-exponential burst (equation 2 shown below) by non-linear regression analysis using the KinTek Explorer software and the burst parameters $k_{\text {burst }}$ (decay rate; eigenvalue) steadystate $k_{\text {cat }}$ and the amplitude $(A)$ were calculated (Table $\mathbf{S 1}$ below).

Substrate concentration dependent experiments were performed using OH-KYN and KYN for HsKYNase and PfKYNase respectively. The enzyme ( $\sim 1 \mu \mathrm{M}$ final concentration after mixing with the substrate) and the substrate were mixed in the stopped-flow apparatus at $37^{\circ} \mathrm{C}$ and the fluorescence was recorded for 0.5 sec. Traces represent the average of at least five runs at each concentration. Stopped-flow fluorescence traces were initially fit to analytical single burst function by non-linear regression analysis and ultimately all the data were fit globally based on the minimal kinetic models shown in Scheme 1 in the main text for the determination of individual rate constants using the KinTek Explorer software ${ }^{2}$ as described in the following section.

$\mathrm{Y}=\mathrm{A} \mathrm{e}^{\left(-k{ }_{\text {burst }}{ }^{\mathrm{t}}\right)+k_{\text {cat }} \mathrm{t} \quad(\text { equation 2) }}$ 
where $\mathrm{A}$ is the amplitude, $k_{\text {burst }}$ is the decay rate of the burst phase (eigenvalue) and $k_{\text {cat }}$ is the steady-state turnover rate of the reaction.

\section{Global fitting analysis of pre-steady-state data.}

As part of the global optimization process, the data which were collected over a series of substrate concentrations for a given experiment, were scaled based on the reference curve (either for AA or OH-AA) in order all traces to start at $\mathrm{t}=0$ from the same background fluorescence ( $\mathrm{y}$-axis intercept of the standard curve). At the end of the global fitting analysis, the data were scaled using a correction factor $(<5 \%)$ for each trace that accounts for slight lamp fluctuation and drift between the traces. For the numerical integration of the rate equations, the measured fluorescence of either AA or OH-AA was expressed as an observable output function as the sum of the free and enzyme-bound product defined by the generated standard curves. More specifically, the measured fluorescence was expressed as $\mathrm{y}=\mathrm{a} \times(\mathrm{E} \sim \mathrm{OH}-\mathrm{AA}+\mathrm{OH}-$ $\mathrm{AA})+\mathrm{b}$ for the HsKYNase or $\mathrm{y}=\mathrm{a} \times(\mathrm{E} \sim \mathrm{AA}+\mathrm{AA})+\mathrm{b}$ for the PfKYNase, where $a$ is the slope of either the $\mathrm{AA}$ or the OH-AA standard curve and $\mathrm{b}$ is the $\mathrm{y}$-axis intercept of this curve. The species that are experimentally detected by the stopped-flow assay are indicated by red capital letters in Scheme $\mathbf{1}$ in the main text either as AA or OH-AA. The rate constants calculated from the numerical integration analysis are reported in the respective Tables $\mathbf{S 2}$ and $\mathbf{S 3}$ and Scheme 1 while the statistical confidence contour analyses $^{3}$ for the experiments are shown below in Figures S4 and S5. Values shown in green in Scheme 1 in both reaction pathways are the final result of the best fit values of the numerical integration and are also included in the respective tables with their upper and lower limits. ${ }^{4}$ The values in parentheses were held fixed during fitting.

\section{Model development for HsKYNase and PfKYNase reactions with OH-KYN and KYN respectively}

In order to be able to globally fit the data using the KinTek Explorer program a minimal kinetic model is needed. For the concentration-dependent pre-steady-state data for HsKYNase and PfKYNase for the reaction with their preferred substrate we developed a minimal four-step kinetic model for each reaction (where the substrate binds to the enzyme forming the E $\sim S$ complex, followed by the formation of the $\mathrm{E} \sim \mathrm{P} \sim(\mathrm{OH})-\mathrm{AA}$ complex where $\mathrm{P}$ represents the $2^{\text {nd }}$ product ALA, the release of either OH-AA or AA and finally the release of ALA, E + P) based on the following: i) the observation of a burst phase upon mixing of the enzyme with the substrate suggesting that the rate-limiting step is, at least partially, the product release ii) the high steady-state specificity constant $k_{\mathrm{cat}} / \mathrm{K}_{\mathrm{M}}$ for both reactions (Table 1) that sets a lower limit for the second order rate constant for substrate binding $k_{1}$ of the reaction (Scheme 1) iii) the rate of the burst phase (Table 1) provides an approximation for the sum of the $k_{2}, k_{-2}, k_{3} \& k_{-3}$ intrinsic rate 
constants. At this point, it must be emphasized that due to the complexity of the four-step model and the fact that the stopped-flow assay cannot adequately sample the last step of the reaction, the rate constants $k_{4}$ and $k_{-4}$ were not taken into account during the fitting process and thus, there is no values shown in the models in Scheme 1.

Based on the aforementioned information we assigned initial nominal values for the intrinsic rate constants of the first three steps of the four-step minimal kinetic models shown in Scheme 1 and the simulation traces were visually inspected whether they account for the experimental data. Upon finding good initial guesses for the first six rate constants $\left(k_{1}, k_{-1}, k_{2}, k_{-2}, k_{3} \& k_{-3}\right)$, subsequently those that can be defined by the data $\left(k_{2}, k_{-2}, k_{3}\right.$ and $\left.k_{-3}\right)$ were allowed to float whereas those that cannot be defined by the experimental data $\left(k_{1}\right.$ and $k_{-1}$ ) were linked. That is, rate constants $k_{1}$ and $k_{-1}$ were grouped such that only their ratio can be defined and account for the rest of the rate constants. Rate constants $k_{4}$ and $k_{-4}$ describe steps after the first product release (AA and OH-AA) and more specifically the ALA release.

Finally the dataset was subjected to global fitting by numerical integration of the rate equations. During the fitting of the data, it was found that $k_{-2}$ and $k_{-3}$ rate constants could be fixed at zero without affecting the data (largely irreversible steps), whereas when they were larger than zero they were negatively affecting the fit. In addition both models for both enzymes were treated as a rapid equilibrium process $\left(k_{-1}>>k_{2}\right)$ and finally only $k_{1}, k_{2}$ and $k_{3}$ rate constants were allowed to float for the final fit upon fixing rate constant $k_{-1}$ at the values shown in Scheme 1. The final best-fit values for $k_{1} k_{2}$ and $k_{3}$ rate constants were statistically analyzed by confidence contour analysis ${ }^{3}$ to determine whether the calculated values are well-constrained. Indeed, as shown in 1D and 2D confidence contour plots in Figures $\mathbf{S 4}$ and $\mathbf{S 5}$ for HsKYNase and PfKYNase respectively, the estimated values for the rate constants are well constrained by the data with well defined lower and upper limits. In addition, the estimated parameters in both cases of HsKYNase and PfKYNase are slightly correlated as revealed by the elliptical shape of the 2D patterns.

\section{Hydrogen/Deuterium exchange}

Purified HsKYNase and PfKYNase were flash frozen and stored at $-80{ }^{\circ} \mathrm{C}$ in $1 \mathrm{x}$ PBS buffer, pH 7.4. Thawed enzymes were incubated with $5 \mathrm{mM}$ PLP at $37{ }^{\circ} \mathrm{C}$ for at least $3 \mathrm{~h}$. Labeling buffer was generated as 1x PBS in $\mathrm{D}_{2} \mathrm{O}$, pD 7.4, and adding either 3.125 mM KYN, OH-DL-KYN, or no substrate. Following the PLP incubation enzymes were diluted 1:24 (v/v) with labeling buffer at a final concentration of $1.6 \mu \mathrm{M}$ enzyme, $3 \mathrm{mM}$ substrate, and 1x PBS at $37^{\circ} \mathrm{C}$. Enzymes were labeled for 0, 1, 10, or 100 minutes. Limitations due to degradation of OH-DL-KYN meant each time course was repeated in triplicate with freshly prepared label buffer for both substrates. Labeling reactions were quenched 1:1 in $2 \mathrm{M} \mathrm{GuHCl}$, $0.8 \%(\mathrm{v} / \mathrm{v}) \mathrm{FA}$ at $1{ }^{\circ} \mathrm{C}, \mathrm{pH} 2.6$. The samples were injected immediately or flash frozen and thawed prior to online pepsin digestion, trapping, and elution. 


\section{Liquid chromatography}

A Waters M-Class coupled with HDX Manager was used to perform protein digestion with subsequent peptide trapping and elution. Buffers A and B consist of $0.1 \%(\mathrm{v} / \mathrm{v})$ formic acid and $0.1 \%(\mathrm{v} / \mathrm{v})$ formic acid in $100 \%$ acetonitrile, respectively. An Enzymate Pepsin Column (Waters, $300 \AA, 5 \mu \mathrm{m}, 2.1$ x $30 \mathrm{~mm}$ ) was used for online digestion at $15{ }^{\circ} \mathrm{C}$. The HDX manager was equipped with a $50 \mu \mathrm{L}$ loop. Digestion and subsequent trapping were performed for 3 minutes at $100 \mu \mathrm{L} / \mathrm{min}$. All peptides were desalted via reversephase trap (Waters Protein BEH C4 VanGuard Pre-column, 300A, $1.7 \mu \mathrm{m}, 2.1$ x $5 \mathrm{~mm}$ ) and separated on a C18 column (Waters BEH C18 Column, $130 \AA, 1.7 \mu \mathrm{m}, 1 \mathrm{x} 100 \mathrm{~mm}$ ) at $1{ }^{\circ} \mathrm{C}$ using an isocratic gradient from $3-40 \%$ B at $40 \mu \mathrm{L} / \mathrm{min}$ for 7 minutes. Following peptide digestion, a pepsin wash solution, $2 \mathrm{M} \mathrm{GuHCl}$, $4 \% \mathrm{ACN}, 0.8 \% \mathrm{FA}(\mathrm{v} / \mathrm{v})$, was injected to minimize carryover. Blank injections were performed after each sample injection to ensure low carryover was maintained.

\section{Mass spectrometry}

All data were acquired on a Synapt G2-Si Q-TOF (Waters) using positive ion mode in either HDMS or $\mathrm{HDMS}^{\mathrm{E}}$ modes, both of which utilize ion mobility. $\mathrm{HDMS}^{\mathrm{E}}$ mode was used to collect both low (6V) and high energy (ramping $22-44 \mathrm{~V}$ ) data-independent peptide fragmentation data for downstream peptide identification. HDMS mode was used to collect low energy ion data for all deuterated samples. All samples were acquired in resolution mode. Capillary voltage was set to $2.8 \mathrm{kV}$ for the sample sprayer. Desolvation gas was set to $650 \mathrm{~L} /$ hour at $175^{\circ} \mathrm{C}$. The source temperature was set to $80^{\circ} \mathrm{C}$. Cone and nebulizer gas was flowed at $90 \mathrm{~L} /$ hour and 6.5 bar, respectively. The sampling cone and source offset were both set to $30 \mathrm{~V}$. Data were acquired at a scan time of $0.4 \mathrm{~s}$ with a range of 100-2000 m/z. Mass correction was done using $\left[\mathrm{Glu}^{1}\right]$-fibrinopeptide B as a reference mass.

\section{Hydrogen/Deuterium exchange data analysis}

The raw files were exported to Protein Lynx Global Server 3.0.2 (Waters) for peptide identification. Low energy, high energy, and intensity thresholds were set to 250, 50, and 750 counts, respectively. Minimum fragment ion matches per peptide was set to 3. Peptide and fragment tolerances were both set to automatic. Missed cleavages was set to 1 with a false discovery rate of 4 . The PLGS peptide lists were then exported to DynamX 3.0 (Waters). The peptide lists were used to locate peptides in raw files and assign their weighted relative uptake profiles. DynamX 3.0 thresholds were set to 0.3 for Minimum products per amino acid and 1 Minimum consecutive products. The file threshold was set to 3, utilizing peptide lists from a total of 6 controls. All other DynamX 3.0 parameters were left unmodified. All DynamX 3.0 results were 
manually verified and filtered by applying a Welch's t-test at $99 \%$ confidence to identify significant peptides.

Table S1. Burst experiment parameters of the HsKYNase reaction with OH-KYN and PfKYNase with KYN.

\begin{tabular}{|c|c|c|c|}
\hline [Enzyme] & $\boldsymbol{k}_{\text {burst }}\left(\mathbf{s}^{-1}\right)$ & $\boldsymbol{k}_{\text {cat }}\left(\mathbf{s}^{-1}\right)$ & Burst Amplitude $(\boldsymbol{A})$ \\
\hline HsKYNase 7.5 $\mu \mathrm{M}$ & $17.1 \pm 0.11$ & $2.55 \pm 0.1$ & 0.47 \\
\hline HsKYNase 12.5 $\mu \mathrm{M}$ & $18.5 \pm 0.1$ & $2 \pm 0.15$ & 0.43 \\
\hline HsKYNase 17.5 $\mu \mathrm{M}$ & $21 \pm 0.1$ & $1.85 \pm 0.2$ & 0.46 \\
\hline PfKYNase 5 $\mu \mathrm{M}$ & $32 \pm 0.6$ & $5.7 \pm 0.02$ & 0.3 \\
\hline
\end{tabular}

Table S2. Rate constants derived from the global data analysis of the reaction of HsKYNase with OH-KYN.

\begin{tabular}{|c|c|c|c|c|}
\hline Rate & Lower Limit $^{a}$ & Upper Limit $^{b}$ & \% Range & Best Fit \\
\hline$k_{1}$ & 0.944 & 1.15 & 9.9 & $1.04 \mu \mathrm{M}^{-1} \mathrm{~s}^{-1}$ \\
\hline$k_{2}$ & 18.1 & 22.5 & 10 & $20.1 \mathrm{~s}^{-1}$ \\
\hline$k_{3}$ & 7.24 & 8.57 & 8.5 & $7.85 \mathrm{~s}^{-1}$ \\
\hline
\end{tabular}

The data shown in Figure 1B were fit globally to the mechanism shown in Scheme 1 in the main Text. ${ }^{a}$ ${ }^{b}$ Lower and upper limits represent a threshold of 5\% deviation from the minimal Sum Square Error (SSE) in the confidence contours. FitSpace confidence contours are shown in Figure $\mathbf{S} 4$ below. ${ }^{c}$ The percentage range was calculated by dividing the mean of the range by the best fit values as follows: (upper-lower)/( 2 $\times$ best fit). This represents the allowable variation of each best fit value as a percentage. The lower and upper limits as well as the $\%$ range were calculated in the same way for all Tables that present similar data in this study. 
Table S3. Rate constants derived from the global data analysis of the reaction of PfKYNase with KYN.

\begin{tabular}{|c|c|c|c|c|}
\hline Rate & Lower Limit $^{a}$ & Upper Limit $^{b}$ & \% Range & Best Fit \\
\hline$k_{1}$ & 0.89 & 1.14 & 12.5 & $1 \mu \mathrm{M}^{-1} \mathrm{~s}^{-1}$ \\
\hline$k_{2}$ & 31.3 & 41.6 & 14.4 & $35.8 \mathrm{~s}^{-1}$ \\
\hline$k_{3}$ & 11.7 & 13.5 & 7.2 & $12.5 \mathrm{~s}^{-1}$ \\
\hline
\end{tabular}

The data shown in Figure 1E were fit globally to the mechanism shown in Scheme 1 in the main Text. ${ }^{a}$ ${ }^{b}$ Lower and upper limits represent a threshold of 5\% deviation from the minimal Sum Square Error (SSE) in the confidence contours. FitSpace confidence contours are shown in Figure $\mathbf{S 5}$ below. ${ }^{c}$ The percentage range was calculated by dividing the mean of the range by the best fit values as follows: (upper-lower)/( 2 $\times$ best fit). This represents the allowable variation of each best fit value as a percentage. The lower and upper limits as well as the \% range were calculated in the same way for all Tables that present similar data in this study.

Tables S4 and S5 are provided as separate Excel files. 
Table S6. Composition of main culture medium.

\begin{tabular}{|c|c|}
\hline Compound & Concentration \\
\hline Yeast Extract & $20 \mathrm{~g} / \mathrm{L}$ \\
\hline $\mathrm{NH}_{4} \mathrm{Cl}$ & $1 \mathrm{~g} / \mathrm{L}$ \\
\hline $\mathrm{Na}_{2} \mathrm{HPO}_{4} \cdot 12 \mathrm{H}_{2} \mathrm{O}$ & $9 \mathrm{~g} / \mathrm{L}$ \\
\hline $\mathrm{Na}_{2} \mathrm{PO}_{4} \cdot 12 \mathrm{H}_{2} \mathrm{O}$ & $3 \mathrm{~g} / \mathrm{L}$ \\
\hline $\mathrm{D}-\mathrm{Glucose}$ & $20 \mathrm{~g} / \mathrm{L}$ \\
\hline $\mathrm{MgSO}_{4} \cdot 7 \mathrm{H}_{2} \mathrm{O}$ & $1 \mathrm{~g} / \mathrm{L}$ \\
\hline $\mathrm{MnCl}_{2} \cdot 4 \mathrm{H}_{2} \mathrm{O}$ & $0.006 \mathrm{~g} / \mathrm{L}$ \\
\hline $\mathrm{FeSO}_{4} \cdot 7 \mathrm{H}_{2} \mathrm{O}$ & $0.01 \mathrm{~g} / \mathrm{L}$ \\
\hline $\mathrm{Cam}$ & $0.034 \mathrm{~g} / \mathrm{L}$ \\
\hline $\mathrm{ddH}_{2} \mathrm{O}$ & Up to $0.9 \mathrm{~L}$ \\
\hline
\end{tabular}

Table S7. Composition of feeding medium.

\begin{tabular}{|c|c|}
\hline Compound & Concentration \\
\hline${ }^{a}$ D-Glucose & $423 \mathrm{~g} / \mathrm{L}$ \\
\hline${ }^{b}$ Yeast Extract & $144 \mathrm{~g} / \mathrm{L}$ \\
\hline${ }^{a} \mathrm{MgSO}_{4} \cdot 7 \mathrm{H}_{2} \mathrm{O}$ & $13 \mathrm{~g} / \mathrm{L}$ \\
\hline $\mathrm{Cam}$ & $0.034 \mathrm{~g} / \mathrm{L}$ \\
\hline $\operatorname{ddH}_{2} \mathrm{O}$ & Up to $700 \mathrm{~mL}$ \\
\hline
\end{tabular}

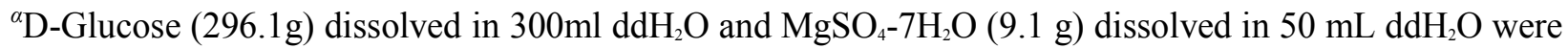
prepared and separately autoclaved.

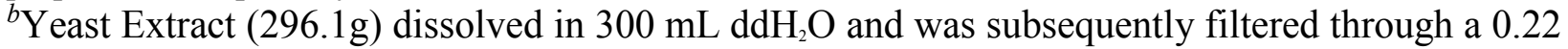
$\mu \mathrm{m}$ filter.

All of the solutions were mixed together, giving a final volume of $\sim 700 \mathrm{~mL}$ whereas Cam was added at a concentration of $34 \mathrm{mg} / \mathrm{L}$. 


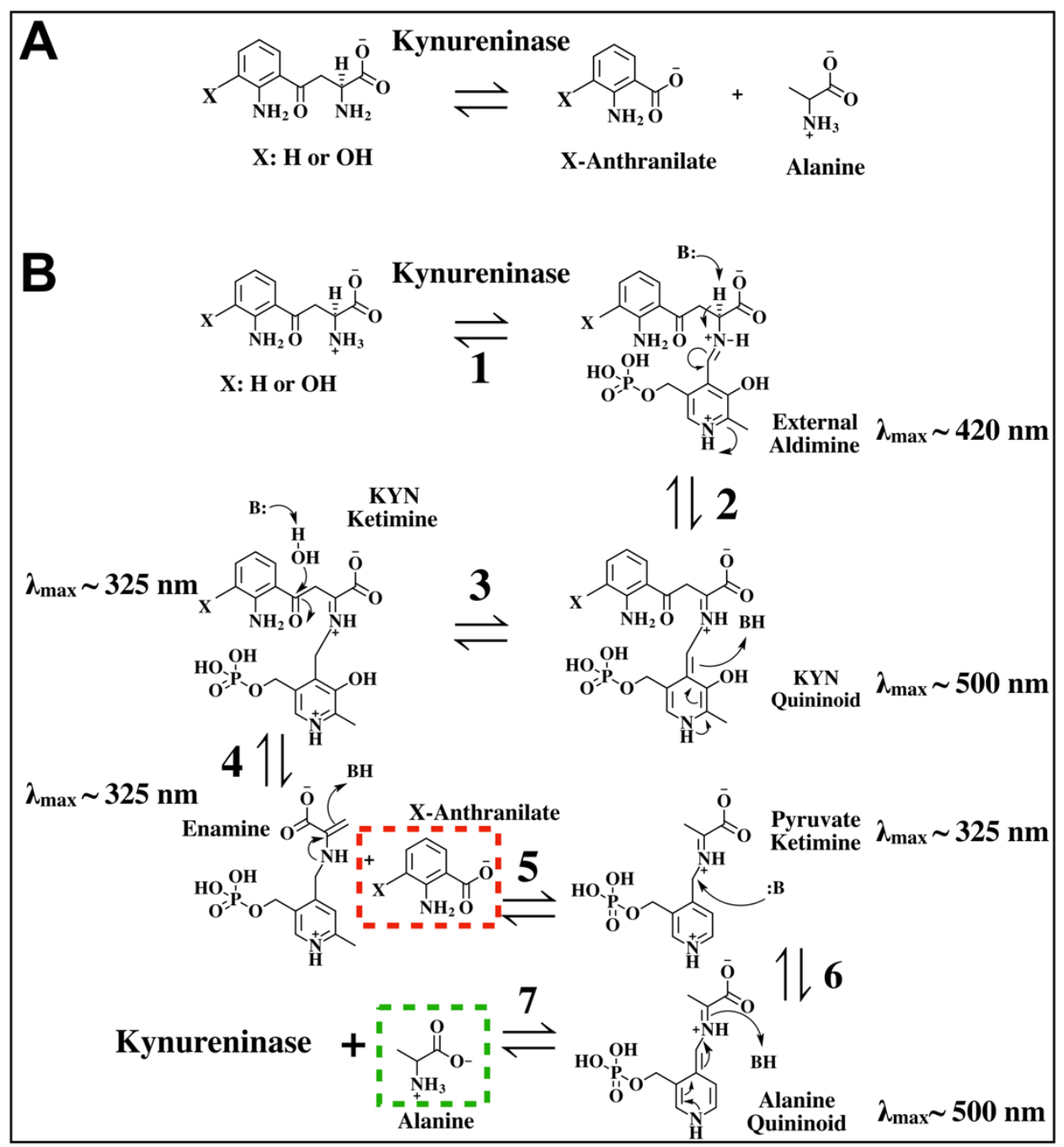

Scheme S1. (A) Reactions catalyzed by Kynureninases. (B) Proposed catalytic mechanism of the reactions. Numbers 1-7 describe catalytic steps and conversion of different intermediates during the reaction. The first product $(\mathrm{OH})$-anthranilate and the second product alanine are labeled with a red and green dashed square respectively. Absorbance maxima for each species are also shown. The chemical structures were generated by ChemDoodle 3D program (iChemLabs). 

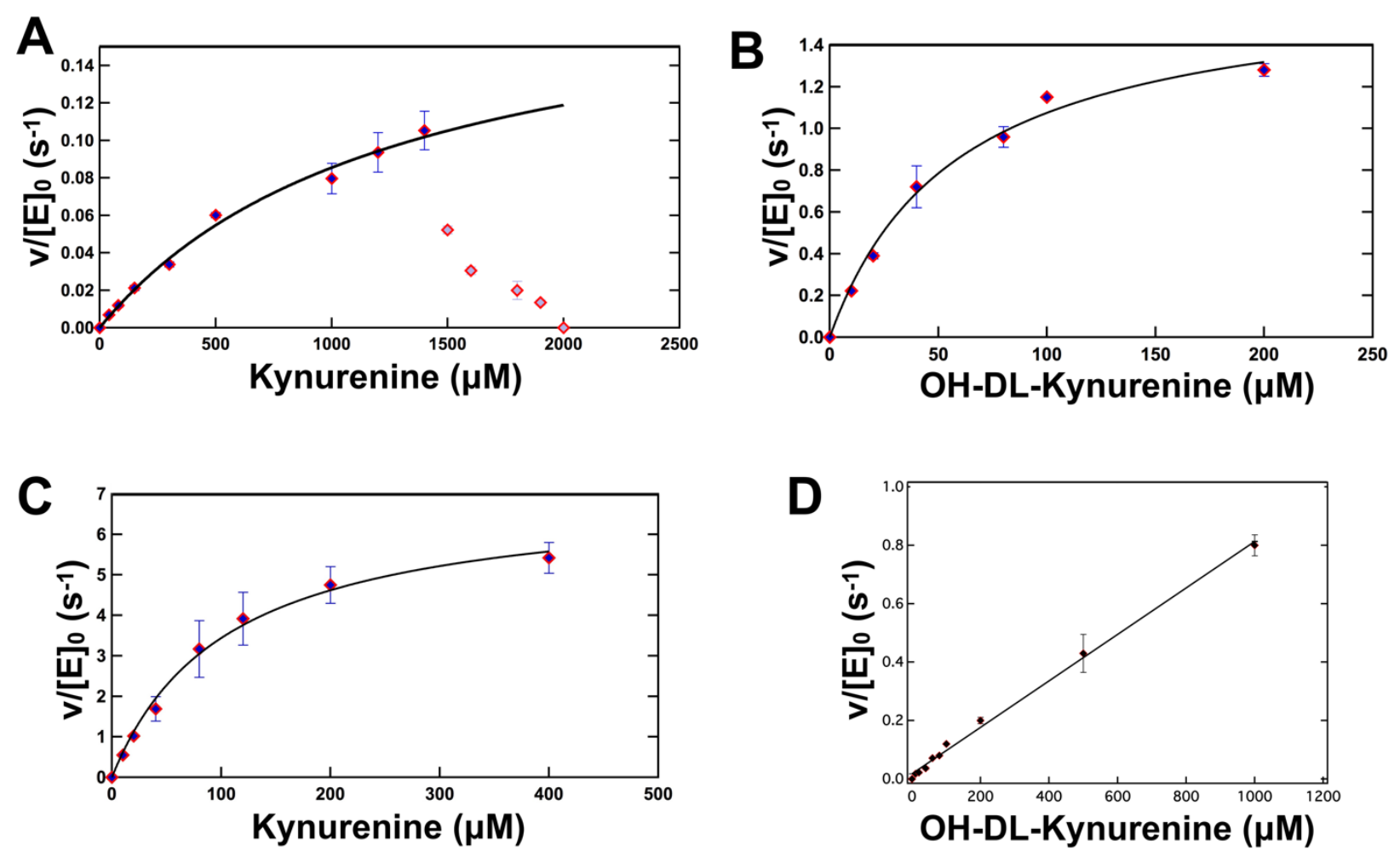

Figure S1. Steady-state kinetic plots for the reaction of hsKYNase with (A) KYN and (B) OH-L-KYN as well as the reaction of PfKYNase with (C) KYN) and with (D) OH-L-KYN. Note that the last five points in plot A for the reaction of hsKYNase with KYN are not included in the fit. They show the substrate inhibition effect on the initial reaction's velocity. Attempts to fit the data in plot A to the steady-state substrate inhibition model ${ }^{5}$ failed due to the very pronounced inhibition effect. The reactions were performed in PBS, pH 7.4 at $37^{\circ} \mathrm{C}$. Each data point is the mean of three different measurements and the error bars represent one standard deviation. The data were fit to Michaelis-Menten eq. 1 except for the plot $D$ where the date were fit to a straight line $(y=a * x+b)$ and the slope of the line (a) defines the second order rate constant for substrate binding $k_{\mathrm{ca}} / \mathrm{K}_{\mathrm{M}}$. The data analysis was done using the software SoftZymics and Igor Pro (Wavemetrics). 


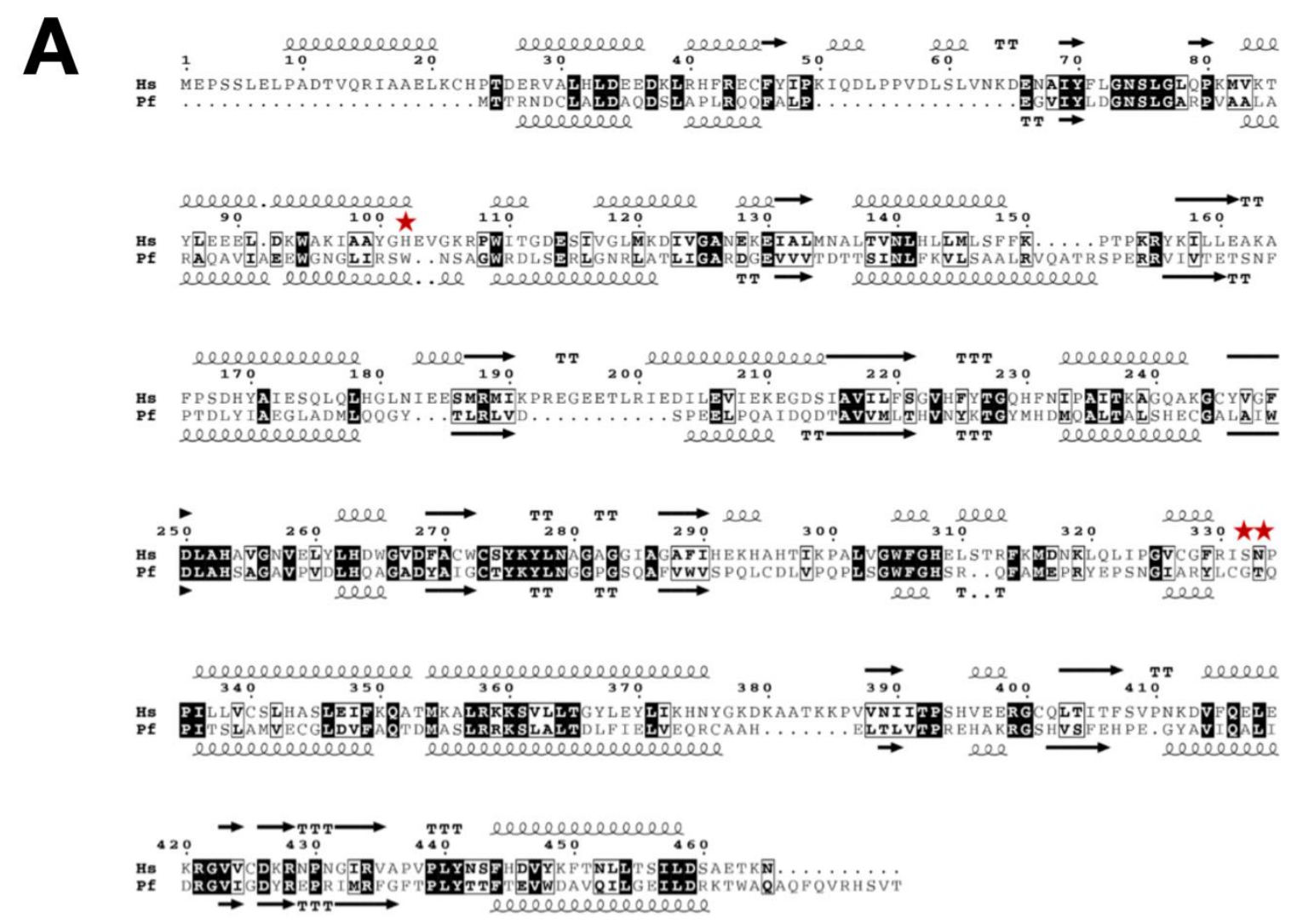

B
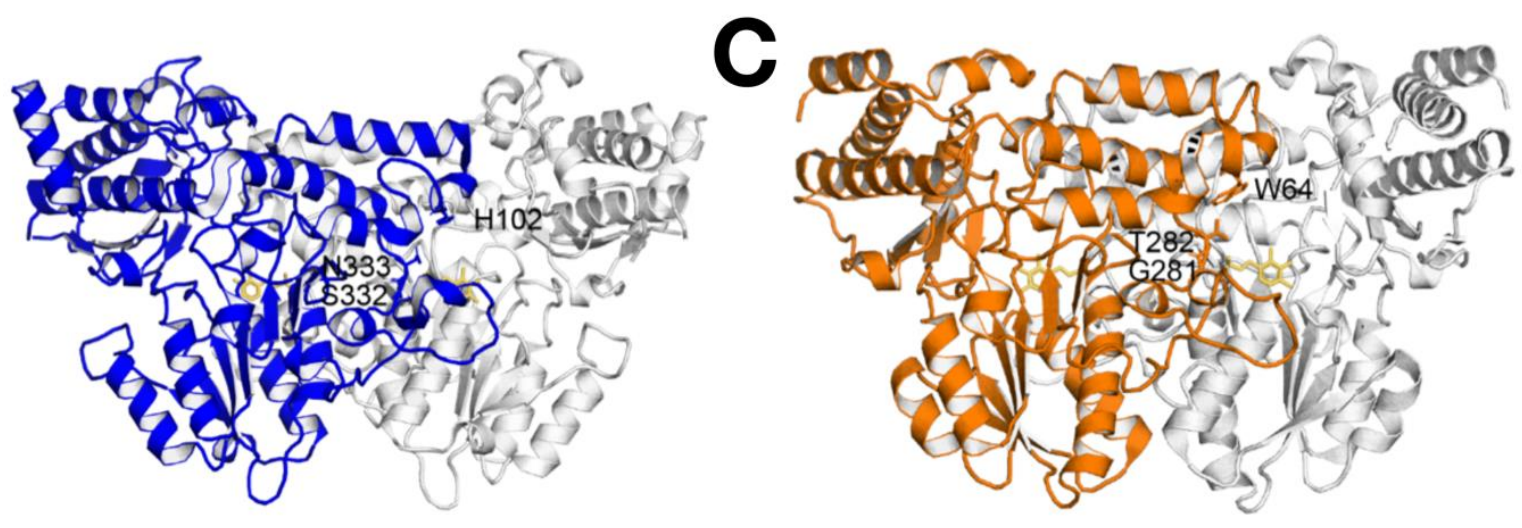

Figure S2. (A) Sequence alignment and secondary structure of Hs and PfKYNase. Conserved residues (shown in black) and similar residues are outlined. First shell triplet residues indicated by red stars. (B) HsKYNase homodimer with the two monomers colored in blue (monomer A) and white (monomer B). (C) PfKYNase homodimer with the two monomers colored in orange (monomer A) and white (monomer B). Triad residues and PLP are shown as either blue or orange and yellow sticks respectively. 


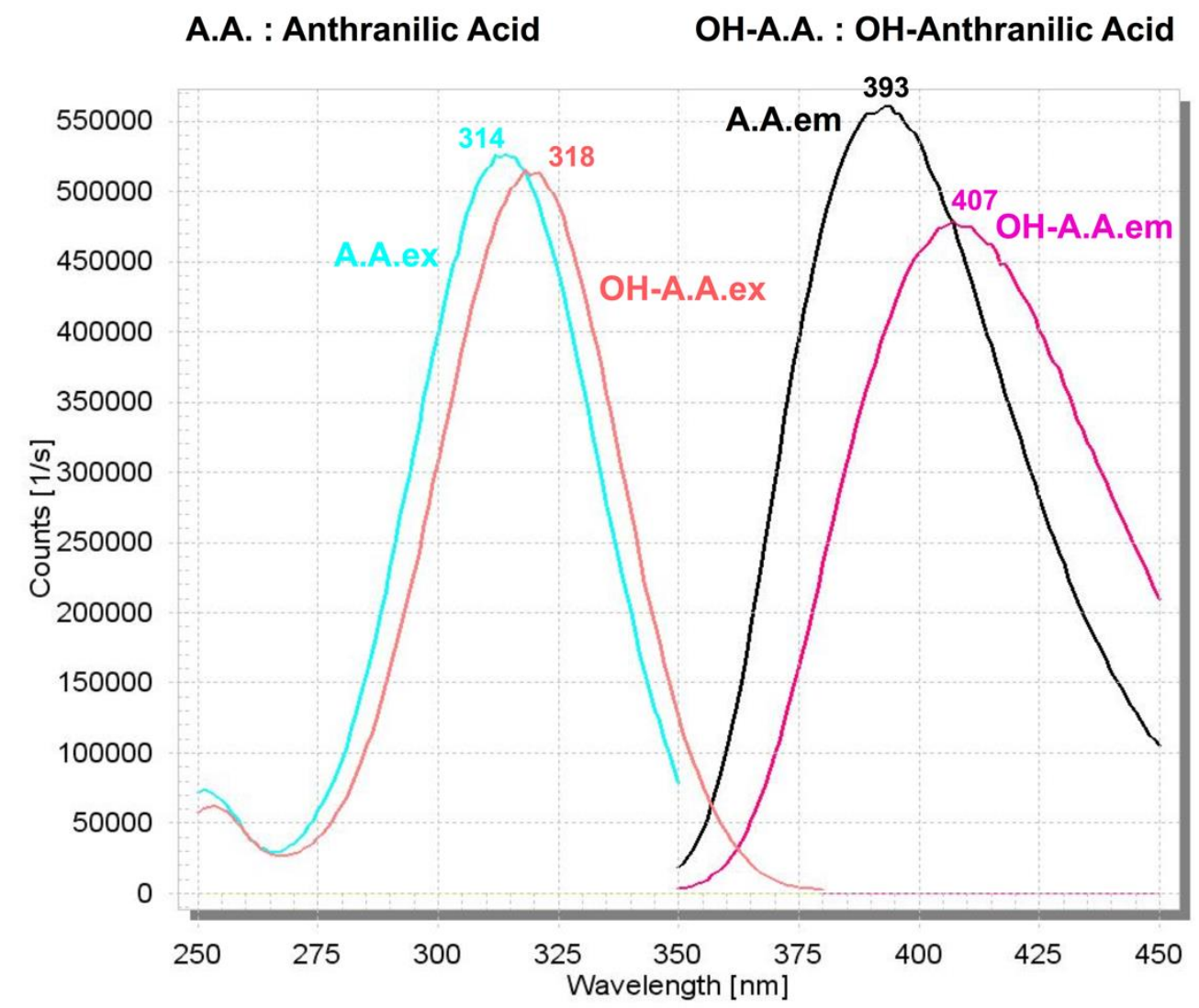

Figure S3. Excitation and emission spectra for AA and OH-AA obtained in PBS, pH 7.4 


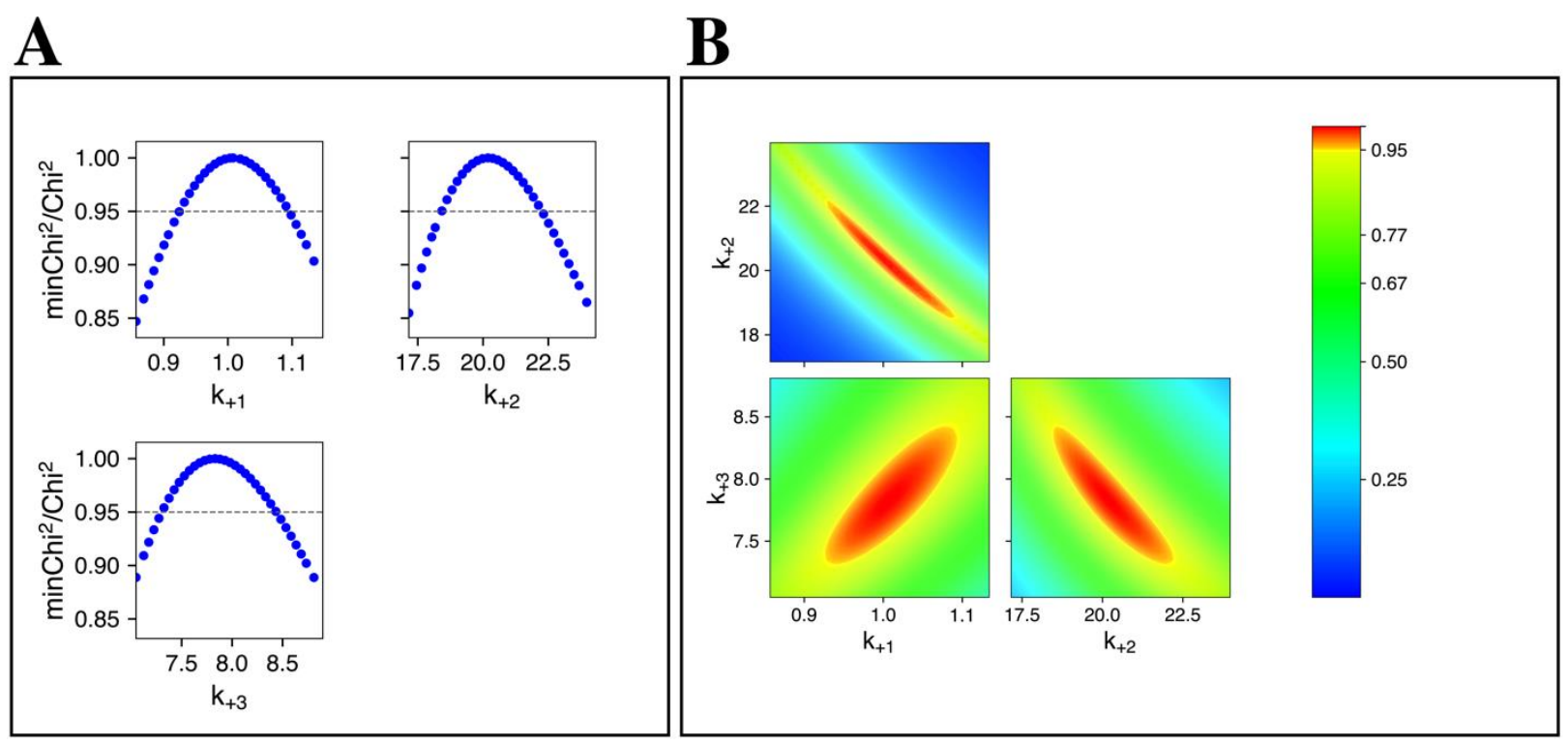

Figure S4. FitSpace confidence contour analysis for the global fit of the kinetic data obtained for the reaction of HsKYNase with OH-KYN. (A) \& (B): 1D and 2D FitSpace analysis respectively for the fit of the rate constants $k_{1}, k_{2}$ and $k_{3}$ (expressed as s ${ }^{-1}$ ) to the model shown in Scheme 1A in the main text. 


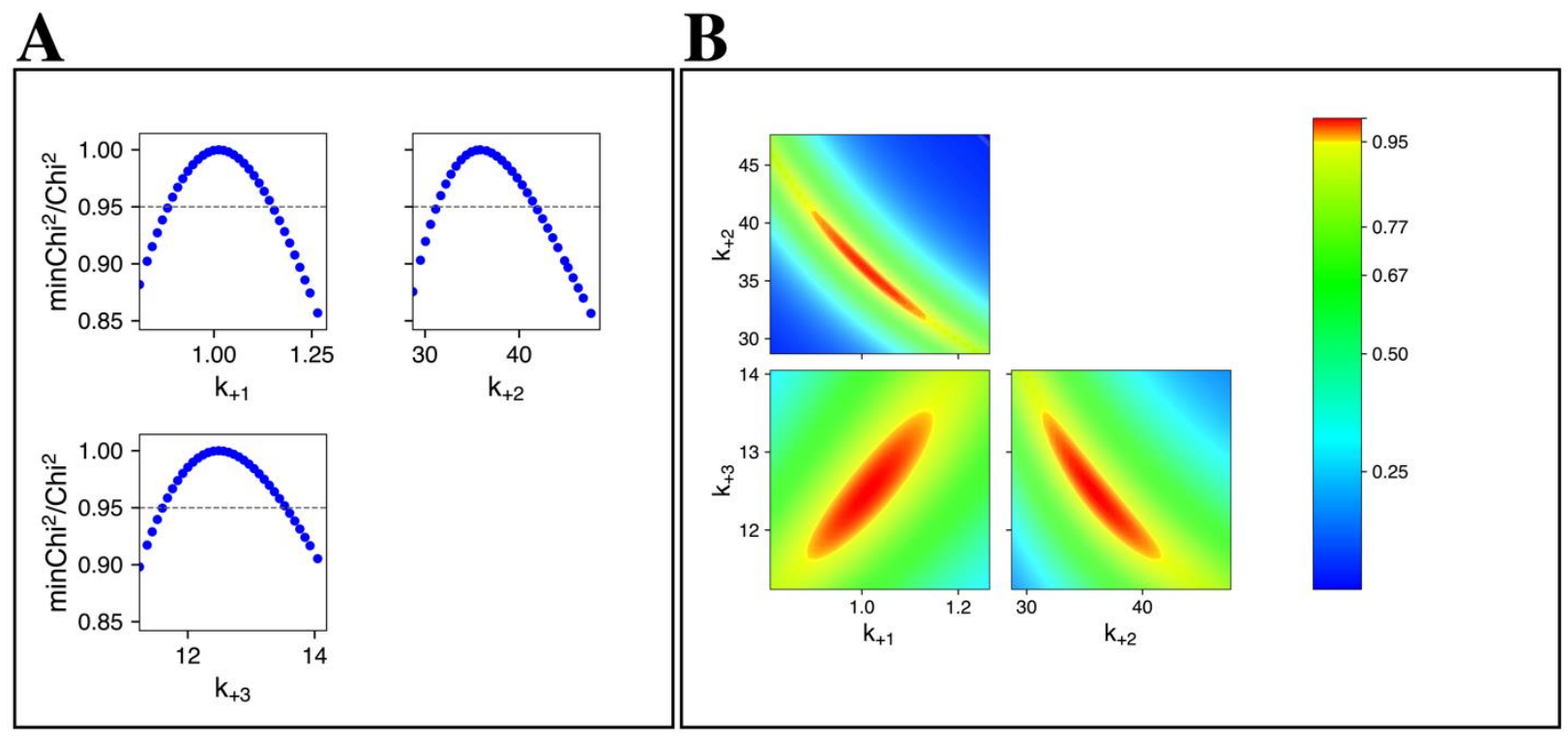

Figure S5. FitSpace confidence contour analysis for the global fit of the kinetic data obtained for the reaction of PfKYNase with KYN. (A) \& (B): 1D and 2D FitSpace analysis respectively for the fit of the rate constants $k_{1}, k_{2}$ and $k_{3}$ (expressed as $\mathrm{s}^{-1}$ ) to the model shown in Scheme 1B in the main text. 
A

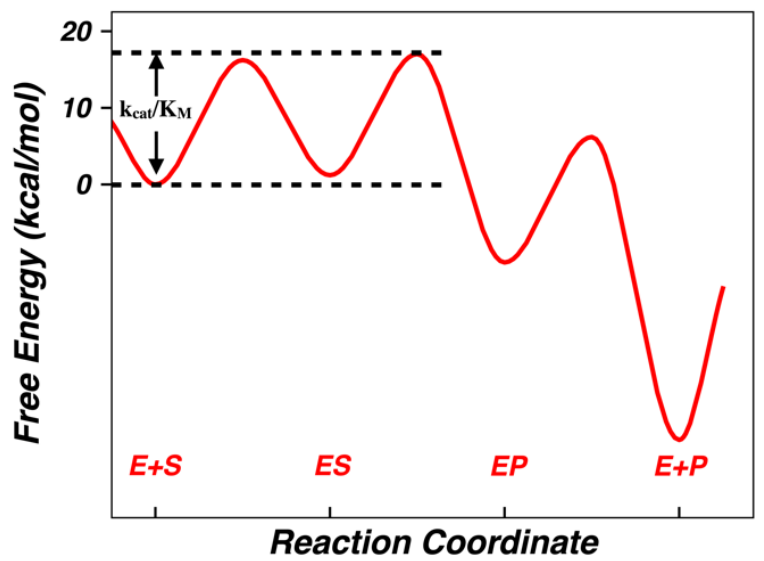

B

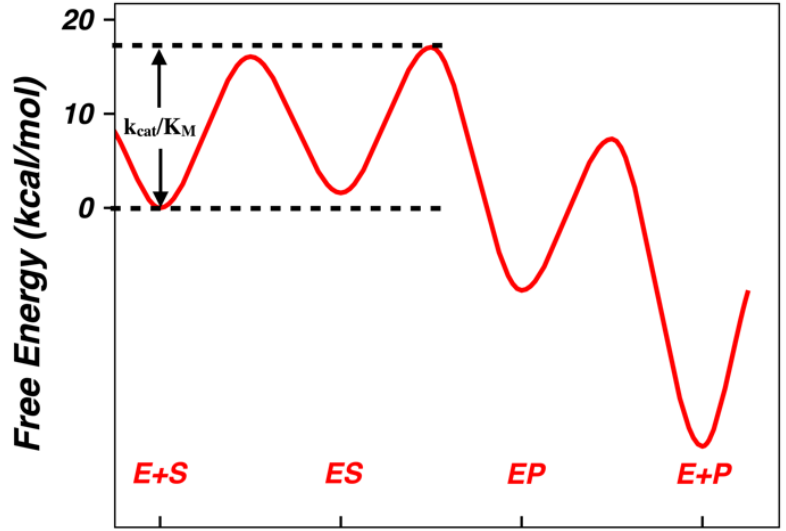

Reaction Coordinate

Figure S6. Free energy profiles for the reaction of (A) HsKYNase with OH-KYN and (B) PfKYNase with KYN respectively shown in Scheme 1 in the main Text. The free energies of activation were calculated from the Eyring equation $\Delta \mathrm{G}^{\ddagger}=-\mathrm{RT} \ln \left(k\left(\alpha \times k_{\mathrm{B}} \mathrm{T} / \mathrm{h}\right)\right)$, where $\alpha$ is a transmission coefficient set at 1 using the KinTek Explorer program. Here the term $\mathrm{P}$ defines the first product of the reaction i.e. either OH-AA or AA. 

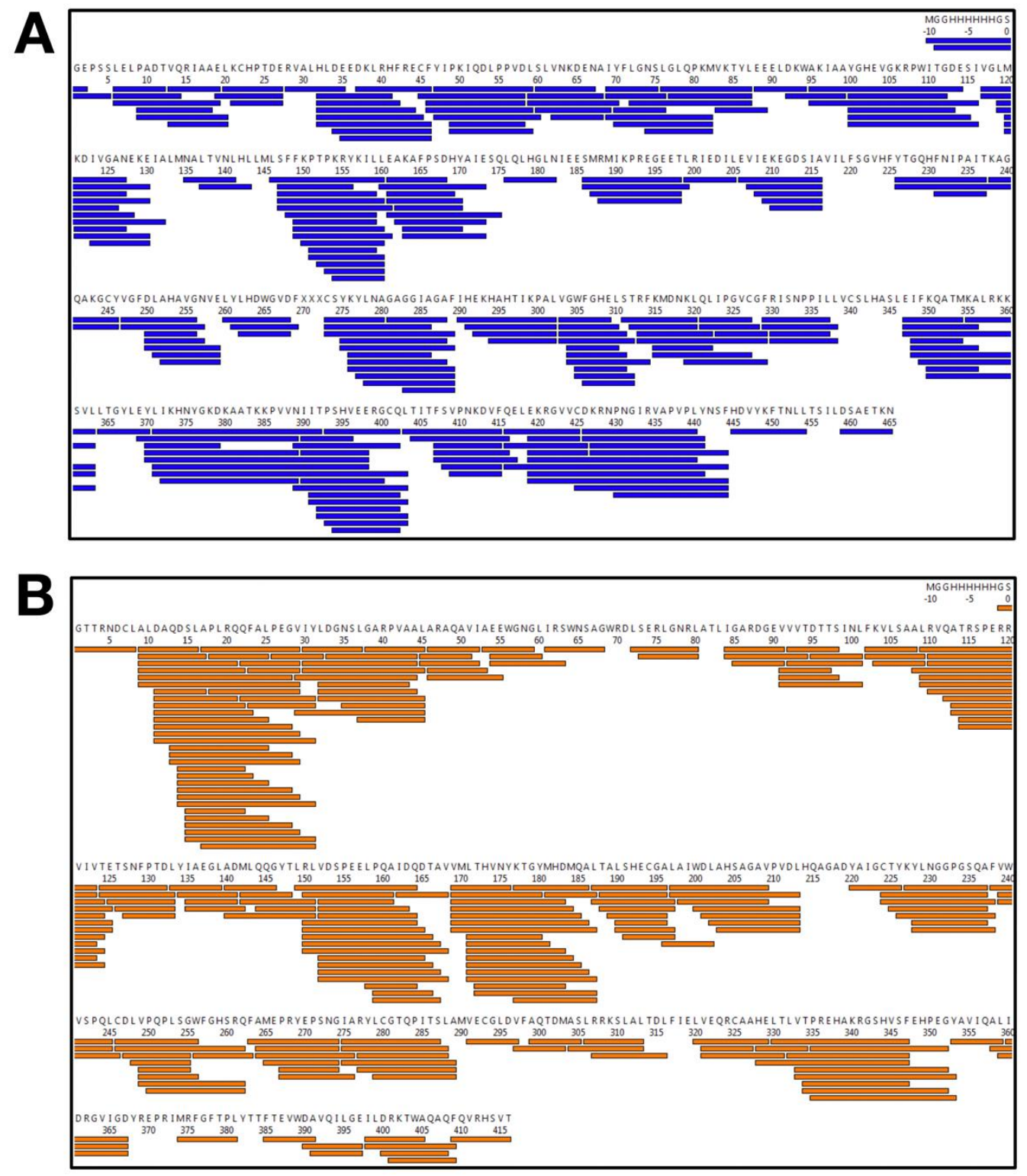

Figure S7. Peptides recovered after digestion and manual refinement of KYNases. (A) HsKYNase coverage map with 183 peptides covering $93.3 \%$ of the protein, averaging 4.45 redundancy per recovered amino acid. (B) PfKYNase coverage map with 189 peptides covering $87.6 \%$ of the protein, averaging 5.77 redundancy per recovered amino acid. 

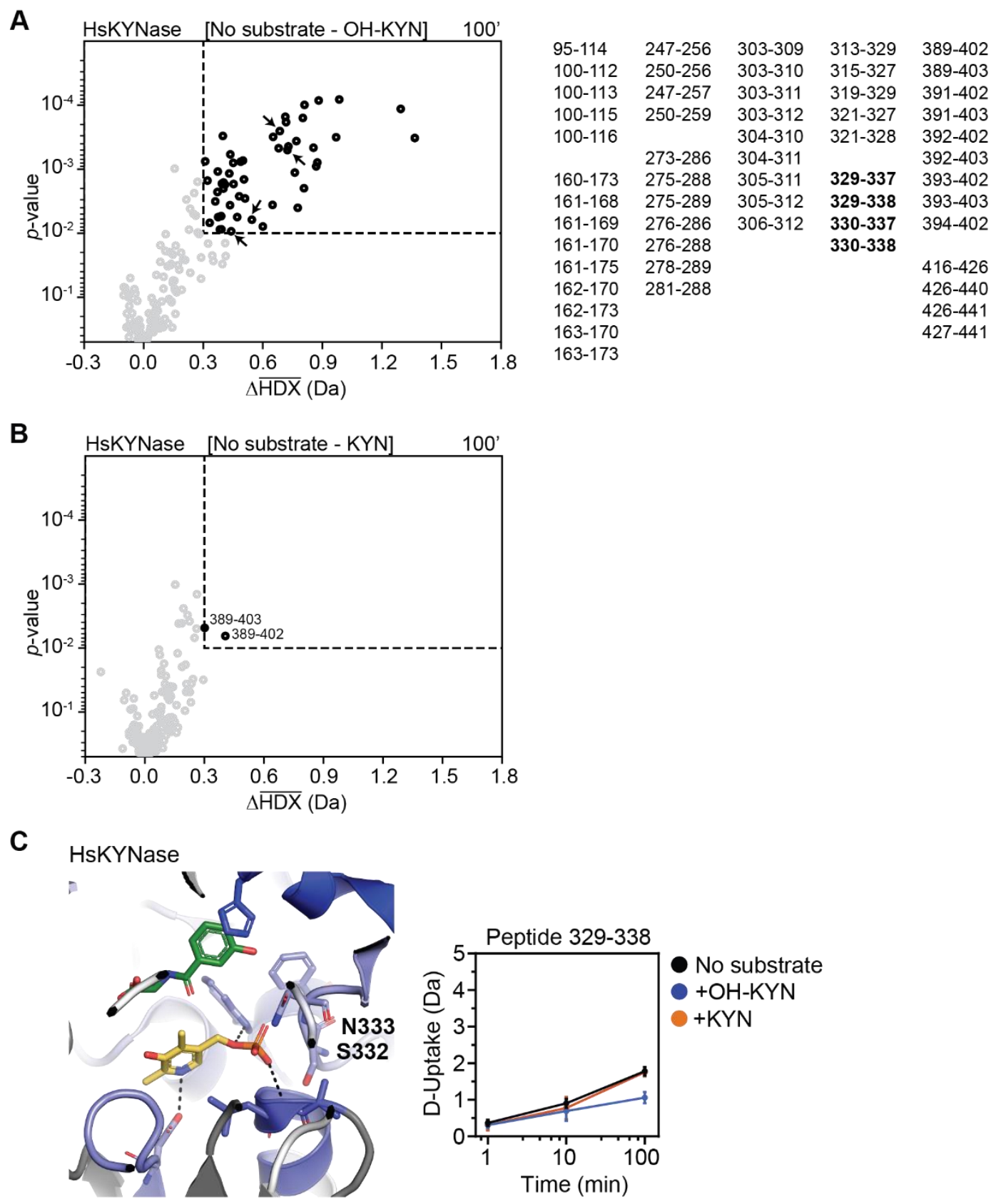

Figure S8. HDX-MS of HsKYNase with OH-KYN (A) or KYN (B). Volcano plots showing the average $\triangle \mathrm{HDX}$ calculated by subtracting either OH-KYN (A) or KYN (C) from no substrate after 100 minutes D exposure. P-values were calculated using a Welch's t-test. Significance cutoffs are p-value $<0.01$ and an average $\Delta \mathrm{HDX}>0.3 \mathrm{Da}$. Significant peptides are shown in black. Boundaries of significant peptides are listed on the right (A) or on the plot (B) and the four bolded peptides are indicated by arrows. (C) HsKYNase active site with example D-uptake plots. D-uptake plot traces are no substrate (black), OH-KYN (blue) or KYN (orange). Y-axis range is $50 \%$ of max Duptake assuming the $\mathrm{N}$-terminal residue undergoes complete back-exchange. Data have not been corrected for back-exchange. Error bars are $\pm 2 \sigma$ from three technical replicates. The structure is depicted as in Figure 3. 

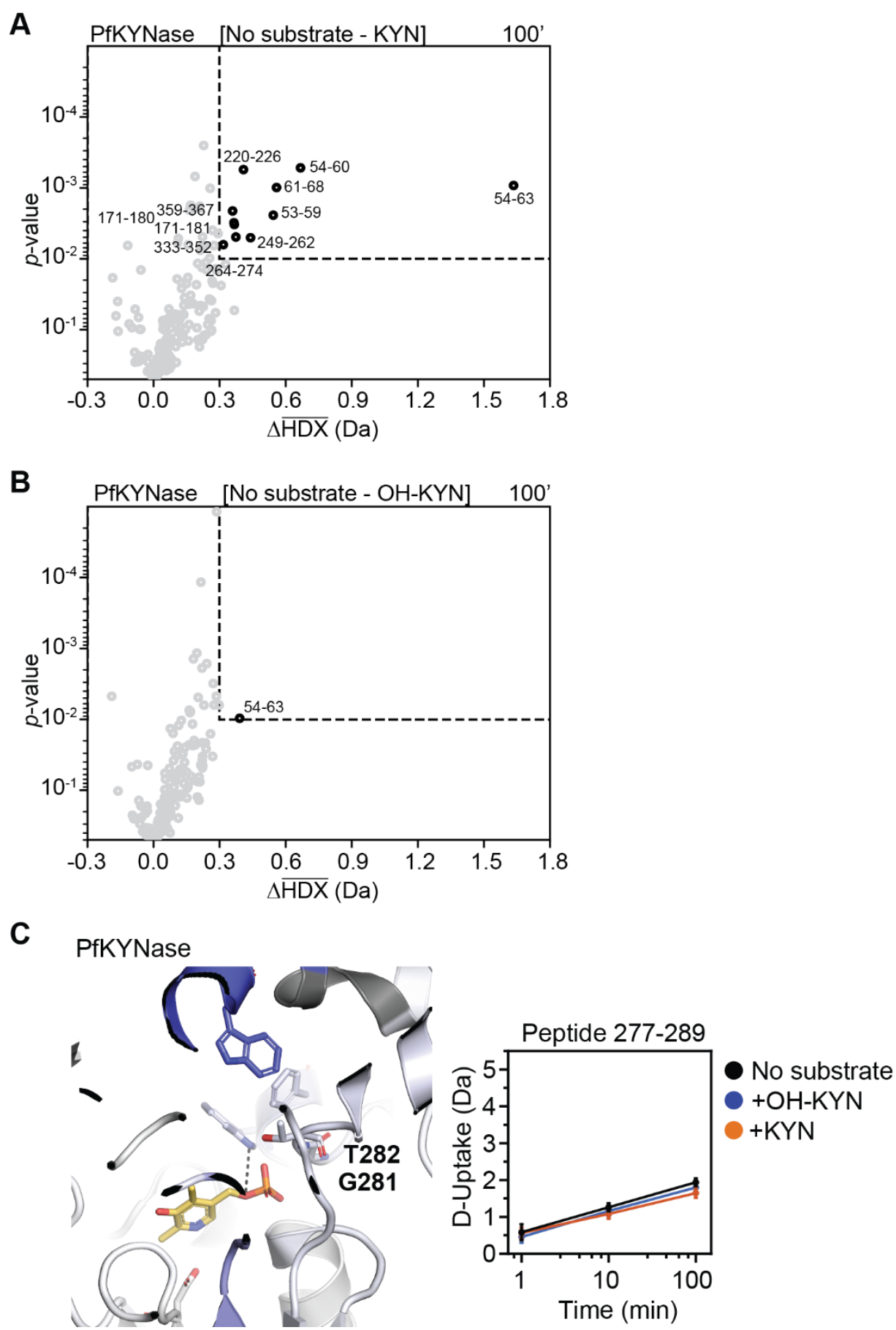

Figure S9. HDX-MS of PfKYNase with KYN (A) or OH-KYN (B). Volcano plots showing the average $\triangle \mathrm{HDX}$ calculated by subtracting either KYN (A) or OH-KYN (C) from no substrate after 100 minutes D exposure. P-values were calculated using a Welch's t-test. Significance cutoffs are p-value $<0.01$ and an average $\Delta \mathrm{HDX}>0.3 \mathrm{Da}$. Significant peptides are shown in black and boundaries of significant peptides are labeled on the plot. (C) PfKYNase active site with example D-uptake plots. D-uptake plot traces are no substrate (black), OH-KYN (blue) or KYN (orange). Y-axis range is 50\% of max D-uptake assuming the N-terminal residue undergoes complete back-exchange. Data have not been corrected for back-exchange. Error bars are $\pm 2 \sigma$ from three technical replicates. The structure is depicted as in Figure 5. 

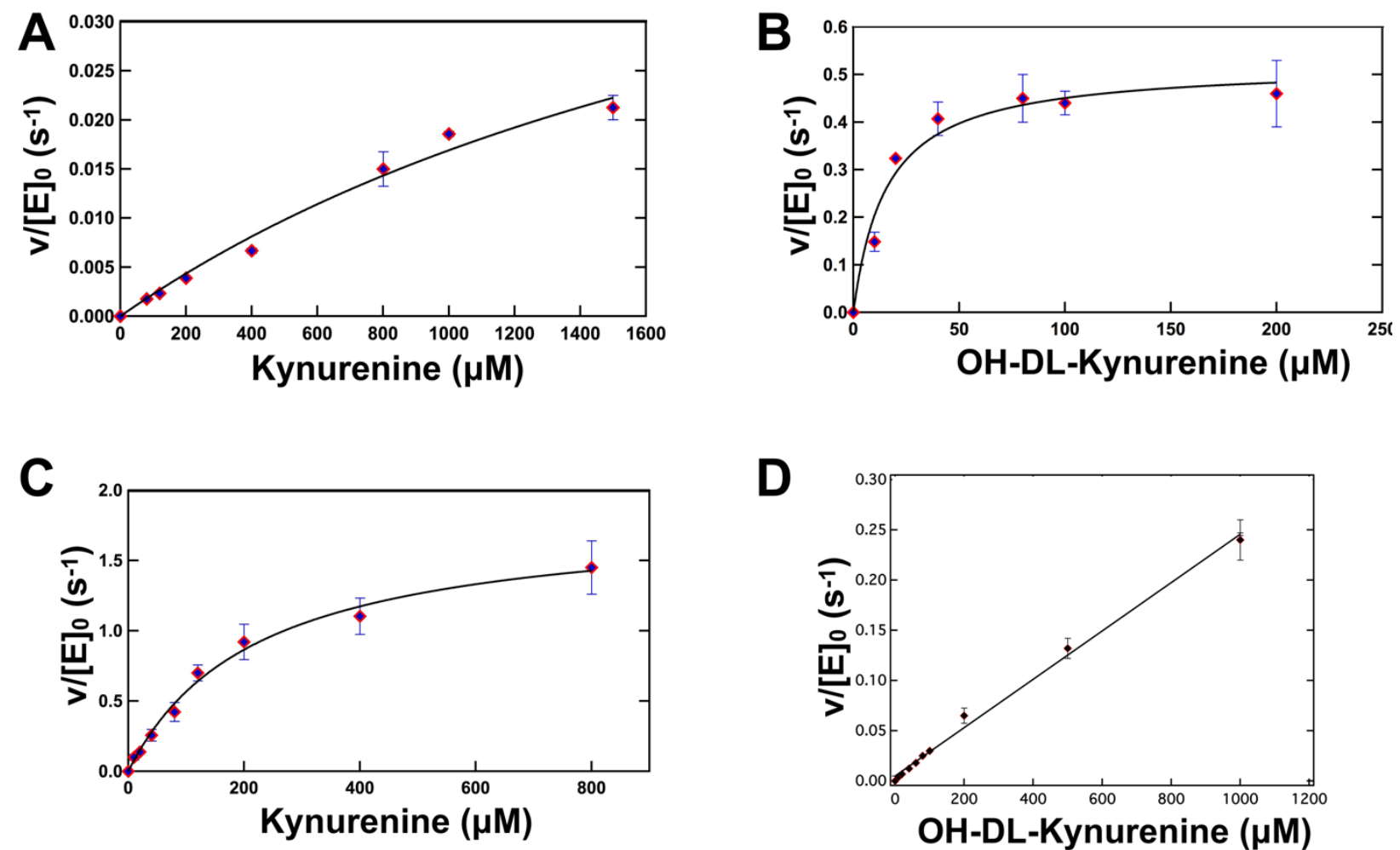

Figure S10. Steady-state kinetic plots for the reaction of HsKYNase with (A) KYN and (B) OH-KYN as well as the reaction of PfKYNase with (C) KYN) and with (D) OH-KYN. The reactions were performed in $\mathrm{D}_{2} \mathrm{O}-\mathrm{PBS}$, pD 7.4 at $37^{\circ} \mathrm{C}$. Each data point is the mean of three different measurements and the error bars represent one standard deviation. The data were fit to Michaelis-Menten eq. 1 except for the plot $\mathrm{D}$ where the date were fit to a straight line $(\mathrm{y}=\mathrm{a} * \mathrm{x}+\mathrm{b})$ and the slope of the line (a) defines the second order rate constant for substrate binding $k_{\text {cat }} / \mathrm{K}_{\mathrm{M}}$. The data analysis was done using the software SoftZymics and Igor Pro (Wavemetrics). 


\section{REFERENCES}

(1) Triplett, T. A.; Garrison, K. C.; Marshall, N.; Donkor, M.; Blazeck, J.; Lamb, C.; Qerqez, A.; Dekker, J. D.; Tanno, Y.; Lu, W.-C.; Karamitros, C. S.; Ford, K.; Tan, B.; Zhang, X. M.; McGovern, K.; Coma, S.; Kumada, Y.; Yamany, M. S.; Sentandreu, E.; Fromm, G.; Tiziani, S.; Schreiber, T. H.; Manfredi, M.; Ehrlich, L. I. R.; Stone, E.; Georgiou, G. Reversal of Indoleamine 2,3-Dioxygenase-Mediated Cancer Immune Suppression by Systemic Kynurenine Depletion with a Therapeutic Enzyme. Nature Biotechnology 2018, 36 (8), 758-764. https://doi.org/10.1038/nbt.4180.

(2) Johnson, K. A.; Simpson, Z. B.; Blom, T. Global Kinetic Explorer: A New Computer Program for Dynamic Simulation and Fitting of Kinetic Data. Anal. Biochem. 2009, 387 (1), 20-29. https://doi.org/10.1016/j.ab.2008.12.024.

(3) Johnson, K. A.; Simpson, Z. B.; Blom, T. FitSpace Explorer: An Algorithm to Evaluate Multidimensional Parameter Space in Fitting Kinetic Data. Anal. Biochem. 2009, 387 (1), 30-41. https://doi.org/10.1016/j.ab.2008.12.025.

(4) Huddleston, J. P.; Schroeder, G. K.; Johnson, K. A.; Whitman, C. P. A Pre-Steady State Kinetic Analysis of the AY60W Mutant of Trans-3-Chloroacrylic Acid Dehalogenase: Implications for the Mechanism of the Wild-Type Enzyme. Biochemistry 2012, 51 (46), 9420-9435. https://doi.org/10.1021/bi3010686.

(5) Yoshino, M.; Murakami, K. Analysis of the Substrate Inhibition of Complete and Partial Types. SpringerPlus 2015, 4 (1), 292. https://doi.org/10.1186/s40064-015-1082-8. 